\title{
Desempenho e oportunidade motora em lactentes com e sem microcefalia: Estudo
}

\section{caso-controle}

\author{
Performance and opportunity of motor stimulation in infants with and without microcephaly: \\ Case-control study
}

Rendimiento y oportunidad de la estimulación motora en bebés con y sin microcefalia: estudio de

Casos y controles

Recebido: 20/05/2021 | Revisado: 28/05/2021 | Aceito: 30/05/2021 | Publicado: 14/06/2021

\author{
Emanuele Mariano de Souza Santos \\ ORCID: https://orcid.org/0000-0002-6130-8979 \\ Universidade Federal de Sergipe, Brasil \\ Universidade Estadual de Ciências da Saúde de Alagoas, Brasil \\ E-mail: manumsouza@yahoo.com.br \\ Íkaro Daniel Carvalho Barreto \\ ORCID: https://orcid.org/0000-0001-7253-806X \\ Universidade Federal Rural de Pernambuco. Brasil \\ E-mail: daniel.carvalho.ib@gmail.com \\ Flávia Calheiros da Silva \\ ORCID: https://orcid.org/0000-0001-6413-4153 \\ Universidade Estadual de Ciências da Saúde de Alagoas, Brasil \\ E-mail: flavia_calheiros@hotmail.com \\ Ricardo Queiroz Gurgel \\ ORCID: https://orcid.org/0000-0001-9651-3713 \\ Universidade Federal de Sergipe, Brasil \\ E-mail: ricardoqgurgel@ gmail.com \\ Andréa Monteiro Correia Medeiros \\ ORCID: https://orcid.org/0000-0002-4930-7623 \\ Universidade Federal de Sergipe, Brasil \\ E-mail: andreamcmedeiros@gmail.com
}

\begin{abstract}
Resumo
O estudo objetivou caracterizar o desempenho motor grosso e as oportunidades de estimulação motora presentes no ambiente domiciliar de lactentes com microcefalia por Zika vírus (ZIKV), comparando-os com lactentes sem microcefalia de instituições públicas de saúde do Nordeste do Brasil, além de verificar o impacto da microcefalia e fatores associados nos desfechos avaliados. Estudo caso-controle que foram utilizados dados de nascimento dos lactentes, gestacionais e sociodemográficos maternos, e dos testes: Gross Motor Function Measure (GMFM), Affordances in the Home Environment for Motor Development (AHEMD), e Sistema de Classificação da Função Motora Grossa (GMFCS). Para analisar o impacto da microcefalia nos testes associada a fatores sociodemográficos ou clínicos utilizou-se a regressão linear múltipla. Participaram 40 lactentes com microcefalia por ZIKV, com média de idade de 15,3 (3,1) meses; e 40 lactentes sem acometimento, com média de idade de 14,8 $(4,4)$ meses. $85 \%$ dos lactentes com microcefalia por ZIKV classificou no nível V do GMFCS. Diferenças significativas $(p<0,001)$ foram evidenciadas para todas as dimensões do GMFM, para variedade de estimulação, brinquedo motricidade fina e pontuação total do AHEMD, com valores sempre menores nos lactentes com microcefalia por ZIKV. Microcefalia associada a variáveis dos lactentes, maternas e da residência são fatores que podem explicar a pontuação no GMFM $(\mathrm{p}<0,001)$. Os resultados reforçam a necessidade de uma assistência integral, centrada na família, uma vez que a resolutividade dos impactos do ZIKV sobre o desenvolvimento motor e o cuidado prestado pelas famílias é uma questão de saúde pública importante.
\end{abstract}

Palavras-chave: Lactente; Microcefalia; Infecção por Zika vírus; Desempenho psicomotor; Características de residência.

\section{Abstract}

The study aimed to characterize the gross motor performance and motor stimulation opportunities present in the home environment of infants with microcephaly by Zika virus (ZIKV), comparing them with infants without microcephaly from public health institutions in Northeast Brazil, and to verify the impact of microcephaly and associated factors on the outcomes assessed. Case-control study that used infants' birth, gestational, and maternal sociodemographic data, 
and the tests: Gross Motor Function Measure (GMFM), Affordances in the Home Environment for Motor Development (AHEMD), and Gross Motor Function Classification System (GMFCS). Multiple linear regression was used to analyze the impact of microcephaly on tests associated with sociodemographic or clinical factors. Forty infants with microcephaly due to ZIKV, mean age 15.3 (3.1) months, and 40 infants without involvement, mean age 14.8 (4.4) months participated. $85 \%$ of the infants with microcephaly due to ZIKV classified in level V of the GMFCS. Significant differences $(\mathrm{p}<0.001)$ were evidenced for all dimensions of the GMFM, for stimulation variety, fine motricity toy and AHEMD total score, with values always lower in infants with ZIKV microcephaly. Microcephaly associated with infant, maternal and residence variables are factors that may explain the GMFM score $(\mathrm{p}<0.001)$. The results reinforce the need for comprehensive, family-centered care, since the resolutivity of ZIKV impacts on motor development and care provided by families is an important public health issue.

Keywords: Infant; Microcephaly; Zika virus infection; Psychomotor performance; Residence characteristics.

\section{Resumen}

El estudio tuvo como objetivo caracterizar el desempeño motor grueso y las oportunidades de estimulación motora presentes en el entorno familiar de los bebés con microcefalia por el virus del Zika (ZIKV), comparándolos con los bebés sin microcefalia de las instituciones de salud pública en el noreste de Brasil, y verificar el impacto de la microcefalia y los factores asociados en los resultados evaluados. Estudio de casos y controles que utilizó datos del nacimiento de los bebés, datos sociodemográficos gestacionales y maternos, y las pruebas: Medida de la Función Motora Gruesa (GMFM), Asequibilidad en el Entorno del Hogar para el Desarrollo Motor (AHEMD), y Sistema de Clasificación de la Función Motora Gruesa (GMFCS). Se utilizó la regresión lineal múltiple para analizar el impacto de la microcefalia en las pruebas asociadas a factores sociodemográficos o clínicos. Participaron 40 lactantes con microcefalia debida al ZIKV, con una edad media de 15,3 (3,1) meses; y 40 lactantes sin afectación, con una edad media de 14,8 (4,4) meses. El $85 \%$ de los bebés con microcefalia debida al ZIKV se clasificaron en el nivel V del GMFCS. Se evidenciaron diferencias significativas $(\mathrm{p}<0,001)$ para todas las dimensiones del GMFM, para la variedad de estimulación, el juguete de motricidad fina y la puntuación total del AHEMD, con valores siempre más bajos en los bebés con microcefalia por el ZIKV. La microcefalia asociada a variables infantiles, maternas y de residencia son factores que pueden explicar la puntuación del GMFM ( $<<0,001)$. Los resultados refuerzan la necesidad de una asistencia integral, centrada en la familia, una vez que la resolución de los impactos del ZIKV sobre el desarrollo motor y el cuidado prestado a las familias es una cuestión de salud pública importante.

Palabras clave: Lactante; Microcefalia; Infección por el virus del Zika; Rendimiento psicomotor; Características de la residencia.

\section{Introdução}

Nos anos de 2015 e 2016, uma grave situação de saúde pública foi deflagrada no Brasil, com aumento de casos de microcefalia, causando preocupação na comunidade científica (Baud et al., 2017; Heymann et al., 2016). Evidências da vigilância em saúde, incluindo estudos epidemiológicos, clínicos e laboratoriais comprovaram a associação entre anormalidades do sistema nervoso central (SNC) e a infecção por Zika vírus (ZIKV) (Baud et al., 2017). A descrição de outros achados clínicos no curso de desenvolvimento desses lactentes, como alterações neuromotoras, sensoriais, além de problemas alimentares e gastrointestinais, caracterizou a Síndrome Congênita do Zika vírus (SCZ) (del Campo et al., 2017).

Em geral, essa população apresentava graves danos no desenvolvimento neuropsicomotor (Brunoni et al., 2016), sendo descritos riscos para sequelas como atraso motor, hipertonia, deformidades e contraturas de membros, e paralisia cerebral (PC) (Brunoni et al., 2016; Marques et al., 2019; Pessoa et al., 2018), impactando desfavoravelmente na participação em atividades como o brincar, nos diferentes ambientes, como o domiciliar (Duarte et al., 2019); levando a limitações na capacidade funcional e na qualidade de vida individual e familiar (Brunoni et al., 2016).

No entanto, as manifestações clínicas e as complicações do desenvolvimento decorrentes da ZIKV são complexas e requerem avaliação e acompanhamento dos lactentes acometidos, incluindo suas famílias (Kapogiannis et al., 2017). Considerando que o desenvolvimento motor depende do funcionamento do SNC (Brasil, 2016) e da quantidade e qualidade dos estímulos disponibilizados ao lactente, propiciar ambientes com altas oportunidades de estimulação faz-se necessário (Brasil, 2016; Cunha et al., 2018). O ambiente domiciliar é o primeiro espaço experienciado pelo lactente, sendo permeado pelas interações familiares, além de configurações da casa e disposição de objetos lúdicos variados (Cunha et al., 2018). 
Dados epidemiológicos mostram que a região nordeste do Brasil foi uma das mais afetadas entre 2015 e 2019, e novos casos de SCZ em 2020 foram registados (Brasil, 2020), requerendo esforços para o fortalecimento dos serviços de atenção à saúde na assistência a essa população e suas famílias, em todos os níveis de atenção, cuja estruturação ainda é um desafio, dada a complexidade do tratamento desses lactentes (Santos et al., 2019).

Os serviços públicos de saúde têm importante papel no delineamento de ações e estratégias de enfrentamento das epidemias, e devem considerar o ambiente em que a criança vive para subsidiar o desenvolvimento de políticas, programas e intervenções (Cunha et al., 2018; Folha et al., 2018). Os profissionais da saúde ao recomendar intervenções, devem considerar os aspectos, como por exemplo espaço doméstico, brinquedos, estímulos e atividades que fazem parte da vida da criança, e auxiliar os familiares para a oferta de situações que estimulem o desenvolvimento de habilidades motoras, a promoção do brincar e a interação com o ambiente (Freitas et al., 2013).

Nessa perspectiva, dada a função ordenadora do nível da atenção primária, a atuação dos Núcleos de Apoio à Saúde da Família (NASF) é fundamental, acompanhando o desenvolvimento dessa população e potencializando a contribuição das famílias (Santos et al., 2019) para o desenvolvimento das crianças em seu ambiente natural.

Dada a estreita relação entre desenvolvimento motor e oportunidades de estimulação recebidas, o estudo teve como objetivo caracterizar o desempenho motor grosso e as oportunidades de estimulação motora no ambiente domiciliar de lactentes com microcefalia por ZIKV da região nordeste do Brasil, comparando-os com lactentes sem acometimento, e verificar o impacto da microcefalia e fatores associados nos desfechos avaliados.

\section{Metodologia}

Trata-se de um estudo caso-controle, realizado entre os meses janeiro de 2017 e outubro de 2019, com lactentes de seis a 24 meses de idade atendidos em quatro instituições públicas de saúde referências para os casos de microcefalia por ZIKV dos estados de Alagoas e Sergipe, região Nordeste do Brasil.

Os lactentes foram selecionados conforme agenda para consulta nas instituições referenciadas. Os critérios de inclusão foram: lactentes entre seis e 24 meses de vida, de ambos os sexos, atendidos na rede pública de saúde dos estados de Alagoas e Sergipe. Foram excluídos os lactentes cujos responsáveis recusaram-se em participar da pesquisa, com síndrome genética associada, e os que apresentaram instabilidade funcional/clínica (pós-intervenção cirúrgica ortopédica).

O grupo de lactentes com microcefalia por ZIKV (GM) foi constituído por amostra de conveniência. A população do grupo controle (GC) foi condicionada à quantidade de lactentes do GM incluída no estudo, na relação 1 x 1.

\section{Instrumentos}

A coleta dos dados foi feita por intermédio de formulário pré-definido, sendo extraídas informações do prontuário clínico e da caderneta de saúde dos lactentes, referentes à condição de nascimento (idade gestacional, peso, perímetro cefálico, Apgar nos primeiro e quinto minutos, complicações neonatais), e ainda, dados sociodemográficos e gestacionais maternos (idade, escolaridade, estado civil, ocupação, renda familiar mensal, local de residência, laudo da ultrassonografia, sintomas de infecção por ZIKV na gestação). A idade cronológica dos lactentes foi registrada no momento de realização dos testes.

Foram aplicados os testes Gross Motor Function Measure (GMFM), Affordances in the Home Environment for Motor Development (AHEMD) no GM e GC, e Sistema de Classificação da Função Motora Grossa - Ampliado e Revisto (GMFCS E \& R) no GM.

O Sistema de Classificação da Função Motora Grossa (GMFCS - E \& R) serve para classificar a funcionalidade motora grossa indicando seu nível de habilidade e limitações. É constituído por escala ordinal de cinco níveis, baseada no 
movimento iniciado voluntariamente, com ênfase no sentar, em transferência e mobilidade, que retrata, em ordem decrescente, independência e funcionalidade de crianças com PC, distribuídas em cinco faixas etárias ( 0 a 2 anos, 2 a 4 anos, 4 a 6 anos, 6 a 12 anos e 12 a 18 anos). É esperado determinado desempenho motor em cada um dos níveis por faixa etária (Silva et al., 2010). O GMFCS têm sido utilizado em crianças com microcefalia por ZIKV (Carvalho et al., 2019; Melo et al., 2020).

O Gross Motor Function Measure (GMFM) serve para avaliar a função motora grossa de crianças com PC entre cinco meses e 16 anos de idade. Composto por 88 itens agrupados em cinco dimensões: A) deitar e rolar - 17 itens, B) sentar - 20 itens, C) engatinhar e ajoelhar - 14 itens, D) em pé - 13 itens; e E) andar, correr e pular - 24 itens; pontuados em escala ordinal de quatro pontos, que varia de zero a três, sendo 0 indicado quando a criança não iniciou a tarefa e 3 que a criança completou a tarefa. Itens não testados recebem pontuação 0. A pontuação de cada dimensão é expressa em porcentagem, considerando a pontuação máxima daquela dimensão. A pontuação percentual total é obtida a partir da soma das porcentagens de todas as dimensões e dividida por 5, variando entre 0\% e 100\% (Russel et al., 2015).

O Affordances in the Home Environment for Motor Development (AHEMD) avalia as oportunidades (affordances) presentes no ambiente domiciliar para o desenvolvimento motor, tendo sido traduzido e validado no Brasil (Caçola et al., 2015; Gabbard et al., 2008). No presente estudo foram utilizadas as suas duas versões:

Affordances no Ambiente Domiciliar para o Desenvolvimento Motor - Escala Bebê - AHEMD-IS, para crianças de três a 18 meses de idade. Abrange questões sobre características da criança e da família, seguido por 35 itens, distribuídos em 4 dimensões (espaço físico, variedade de estimulação, brinquedos - motricidade grossa e brinquedos - motricidade fina), além de uma pontuação total. São categorizadas 4 descrições de affordances motoras em domicílio - sistemas de pontuação (menos adequado, moderadamente adequado, adequado, excelente) (Caçola et al., 2015).

Affordances in the Home Environment for Motor Development (AHEMD-SR), para crianças de 18 a 42 meses de idade. Abrange 67 itens sobre características da criança e da família, espaço físico da residência, atividades diárias e brinquedos e materiais existentes na habitação. Os escores totais são expressos em pontuação bruta e padronizada, como também divididos em cinco categorias (espaço interno, espaço externo, variedade de estimulação, material de motricidade fina e material de motricidade grossa). Estas categorias são classificadas em uma escala tipo Likert (muito fraco, fraco, bom e muito bom), e para o escore total, que varia de cinco a 20 pontos (até nove - baixa, 10 a 16 - média e de 17 a 20 - alta) (Gabbard et al., 2008). No presente estudo, foram calculados os resultados utilizando a calculadora on-line disponível no teste e a classificação das categorias foram padronizadas em: menos adequado, moderadamente adequado, adequado, excelente.

Todos os testes foram aplicados pela pesquisadora principal e cada participante foi avaliado individualmente em todos os protocolos uma única vez. Para aplicação do GMFM houve treinamento presencial prévio com instrutora fisioterapeuta qualificada na aplicação do teste. Uma terapeuta ocupacional também recebeu treinamento para análise da aplicação do GMFM e GMFCS nos lactentes com microcefalia por ZIKV. A análise da confiabilidade intra e inter-avaliadores apresentou ótima consistência, com coeficientes de correlação intraclasse (CCI), nos grupos microcefalia variando de 0,97 a 0,95 e grupo comparativo 0,99 .

A partir da identificação dos lactentes com microcefalia por ZIKV, os procedimentos da pesquisa eram explicados para o seu responsável, obtendo-se assinatura do termo de consentimento. Em seguida, os dados dos lactentes e de sua família eram extraídos dos prontuários clínicos, cadernetas de saúde e, eventualmente complementados diretamente com os responsáveis.

O lactentes e seu responsável eram direcionados para a sala de aplicação dos testes, previamente preparada e climatizada, com todos os recursos preconizados (Russel et al., 2015). A avaliação era iniciada pelo GMFCS, seguida pelo GMFM, conforme ordem de apresentação das cinco dimensões, sendo permitida até três tentativas para cada item (Russel et al., 2015), com duração total aproximada de 60 minutos; e por fim o AHEMD, cujo preenchimento ocorria no momento exato 
do exame, auxiliado pela pesquisadora quando os responsáveis eram analfabetos ou semianalfabetos Toda a avaliação foi filmada integralmente, utilizando-se câmera de vídeo digital acoplada em tripé, modelo Canon Powershot A720 Is 8 Mp Zoom Óptico 6x, e zoom digital controlado por um assistente de pesquisa.

Os registros audiovisuais obtidos com a aplicação do GMFM foram posteriormente assistidos, pontuados e calculados em folha de registro, e devidamente transpostos para planilha eletrônica Microsoft Excel, bem como os demais dados coletados. Foi considerado o comportamento motor espontâneo do lactente, a partir do encorajamento verbal ou do uso de brinquedos (Russel et al., 2015).

\section{Aspectos Éticos}

$\mathrm{O}$ estudo segue as diretrizes e normas que regulamentam as pesquisas envolvendo seres humanos, contidos na Resolução no 466/2012 (Conselho Nacional de Saúde, 2013), foi aprovado pelo Comitê de Ética em Pesquisa do Hospital Universitário da Universidade Federal de Sergipe, sob parecer $n^{\circ}$ 2.935.000. Consentimento por escrito informado foi obtido dos responsáveis de todos os lactentes. Os participantes não receberam nenhum fundo ou compensação para participar deste estudo.

\section{Análise Estatística}

As variáveis categóricas foram descritas por meio de frequência absoluta e relativa percentual. As variáveis contínuas foram descritas por meio de média e desvio padrão. As associações entre variáveis categóricas foram testadas por meio dos testes Qui-quadrado com simulações de Monte-Carlo e Exato de Fisher. A aderência das variáveis contínuas a distribuição normal foi testada pelo teste de Shapiro-Wilk, quando confirmada as diferenças nas medidas de tendência central foram testadas por meio do teste $\mathrm{T}$ para amostras independentes, quando não, por meio do teste de Mann-Whitney.

Foi avaliado o impacto da Microcefalia nos testes AHMED e GMFM ajustado para outros possíveis fatores (sociodemográficos ou clínicos) utilizando regressão linear múltipla, sendo apresentados os coeficientes de regressão, erros padrões, coeficientes de regressão padronizados, estatística $\mathrm{T}$, intervalos de confiança dos coeficientes de regressão, medidas de ajuste como $\mathrm{R}^{2}$ e $\mathrm{R}^{2}$ Ajustado, além de testar possíveis violações dos pressupostos de autocorrelação, multicolinearidade e presença de pontos de influência (alavanca ou outliers) (Fox \& Weisberg, 2018). O nível de significância adotado foi de 5\% e o software utilizado foi o R Core Team 2020.

\section{Resultados}

Participaram do estudo 80 lactentes, sendo 40 lactentes do GM com média de idade de 15,3 ( $\mathrm{DP}=3,1$ ) meses, e 40 lactentes do GC com média de idade de 14,8 (DP=4,4) meses.

Ao compararmos GM e GC nas variáveis sociodemográficas, foram observadas diferenças significativas $(\mathrm{p}<0,001)$ em relação a região de residência, com a maioria do GM proveniente do interior $(72,5 \%)$ e GC da capital $(87,5 \%)$ dos estados, e à renda familiar, tendo GM renda familiar menor $\mathrm{R} \$ 1097,1$ ( $\mathrm{DP}=349)$. Em relação às condições maternas, houve diferenças ( $<<0,001$ ) para idade, com mães mais jovens em GM 24,1 anos (DP=5,7); prevalência de ocupação do lar em GM (89,5\%) e de trabalho formal em GC (50\%). Quanto às condições paternas, GM apresentou menor escolaridade (37,5\%) do que os latentes controles $(\mathrm{p}<0,001)$. Todas as demais variáveis não apresentaram diferenças significativas entre grupos (Tabela 1 ). 
Tabela 1. Características sociodemográficas dos lactentes, das mães e das famílias do GM e GC.

\begin{tabular}{|c|c|c|c|}
\hline & & & \\
\hline & Micro & Controle & p-valor \\
\hline Lactentes & & & \\
\hline Região de residência, $n(\%)$ & & & \\
\hline Capital & $11(27,5)$ & $35(87,5)$ & $<0,001^{\mathrm{F}}$ \\
\hline Interior & $29(72,5)$ & $5(12,5)$ & \\
\hline Estado, $n(\%)$ & & & \\
\hline Alagoas & $20(50)$ & $28(70)$ & $0,110^{\mathrm{F}}$ \\
\hline Sergipe & $20(50)$ & $12(30)$ & \\
\hline Sexo, $n(\%)$ & & & \\
\hline Feminino & $22(55)$ & $20(50)$ & $0,823^{\mathrm{F}}$ \\
\hline Masculino & $18(45)$ & $20(50)$ & \\
\hline Idade, $n(\%)$ & & & \\
\hline$<12$ meses & $6(15,0)$ & $9(22,5)$ & $0,603^{Q}$ \\
\hline $12-18$ meses & $24(60,0)$ & $20(50,0)$ & \\
\hline$>18$ meses & $10(25,0)$ & $11(27,5)$ & \\
\hline Idade, Média (DP) & $15,3(3,1)$ & $14,8(4,4)$ & $0,599^{\mathrm{T}}$ \\
\hline Creche/Escola, $n(\%)$ & $0(0)$ & $5(12,5)$ & $0,057^{\mathrm{F}}$ \\
\hline Mães & & & \\
\hline Idade Materna, Média (DP) & $24,1(5,7)$ & $29,3(7,1)$ & $0,001 \mathrm{w}$ \\
\hline Mãe Estado Civil, $n(\%)$ & & & \\
\hline Casado & $10(27)$ & $24(60)$ & $<0,001 \mathrm{Q}$ \\
\hline Solteiro & $10(27)$ & $13(32,5)$ & \\
\hline União Estável & $17(45,9)$ & $3(7,5)$ & \\
\hline Mãe Ocupação, $n(\%)$ & & & \\
\hline Trabalho Formal & $1(2,6)$ & $20(50)$ & $<0,001 \mathrm{Q}$ \\
\hline Do lar & $34(89,5)$ & $17(42,5)$ & \\
\hline Estudante & $3(7,9)$ & $3(7,5)$ & \\
\hline Famílias & & & \\
\hline Renda Familiar, Média (DP) & $1097,1(349)$ & $2387(2352,3)$ & $<0,001^{\mathrm{W}}$ \\
\hline Tipo Domicílio, $n(\%)$ & & & \\
\hline Casa & $33(94,3)$ & $31(77,5)$ & $0,074 \mathrm{Q}$ \\
\hline Apartamento & $1(2,9)$ & $8(20)$ & \\
\hline Outros & $1(2,9)$ & $1(2,5)$ & \\
\hline Adultos Domicílio & $2,3(0,7)$ & $2,4(0,8)$ & $0,380^{\mathrm{W}}$ \\
\hline Crianças Domicílio & $1,5(0,7)$ & $1,6(0,7)$ & $0,514^{\mathrm{W}}$ \\
\hline Quartos Domicílio & $2,1(0,8)$ & $2,3(0,8)$ & $0,253 \mathrm{~W}$ \\
\hline Tempo de Domicílio & $2,6(0,7)$ & $2,7(0,8)$ & $0,545^{\mathrm{W}}$ \\
\hline Escolaridade Pai, $n(\%)$ & & & \\
\hline Sem instrução/ Fundamental incompleto & $12(37,5)$ & $7(17,5)$ & $0,027 \mathrm{Q}$ \\
\hline Fundamental Completo & $3(9,4)$ & $8(20)$ & \\
\hline Médio Completo & $16(50)$ & $16(40)$ & \\
\hline Superior Completo & $1(3,1)$ & $9(22,5)$ & \\
\hline Escolaridade Mãe, $n(\%)$ & & & \\
\hline Sem instrução/ Fundamental incompleto & $9(25,7)$ & $4(10)$ & $0,009 \mathrm{Q}$ \\
\hline Fundamental Completo & $13(37,1)$ & $6(15)$ & \\
\hline Médio Completo & $10(28,6)$ & $19(47,5)$ & \\
\hline Superior Completo & $3(8,6)$ & $11(27,5)$ & \\
\hline
\end{tabular}

Legenda: $\mathrm{n}$ - Frequência absoluta. \% - Frequência relativa percentual. DP - Desvio padrão. W - Teste de Mann-Whitney. T - Teste T para amostras independentes. F - Teste Exato de Fisher. Q - Teste Qui-Quadrado de Pearson com simulações de Monte-Carlo. Fonte: Autores. 
Com relação as características clínicas de nascimento dos lactentes, foram observadas diferenças significativas ( $\mathrm{p}<0,001)$ para GM em relação ao tipo de parto $(53,8 \%$ normal), peso $2664,6 \mathrm{~kg}(\mathrm{DP}=495,9)$, comprimento $45 \mathrm{~cm}(\mathrm{DP}=3)$ e perímetro cefálico 29,4 cm (DP=2). Para as variáveis gestacionais maternas, as mães do GM apresentaram diferenças significativas $(\mathrm{p}<0,001)$ para intercorrências gestacionais de outra natureza e para alterações no exame de ultrassom no terceiro trimestre gestacional (Tabela 2).

Quanto aos sinais e sintomas clínicos maternos por ZIKV, 80\% do GM relataram a ocorrência de exantema durante a gestação, sendo 53,1\% no primeiro trimestre gestacional.

Tabela 2. Características clínicas ao nascimento dos lactentes e gestacionais maternos do GM e GC.

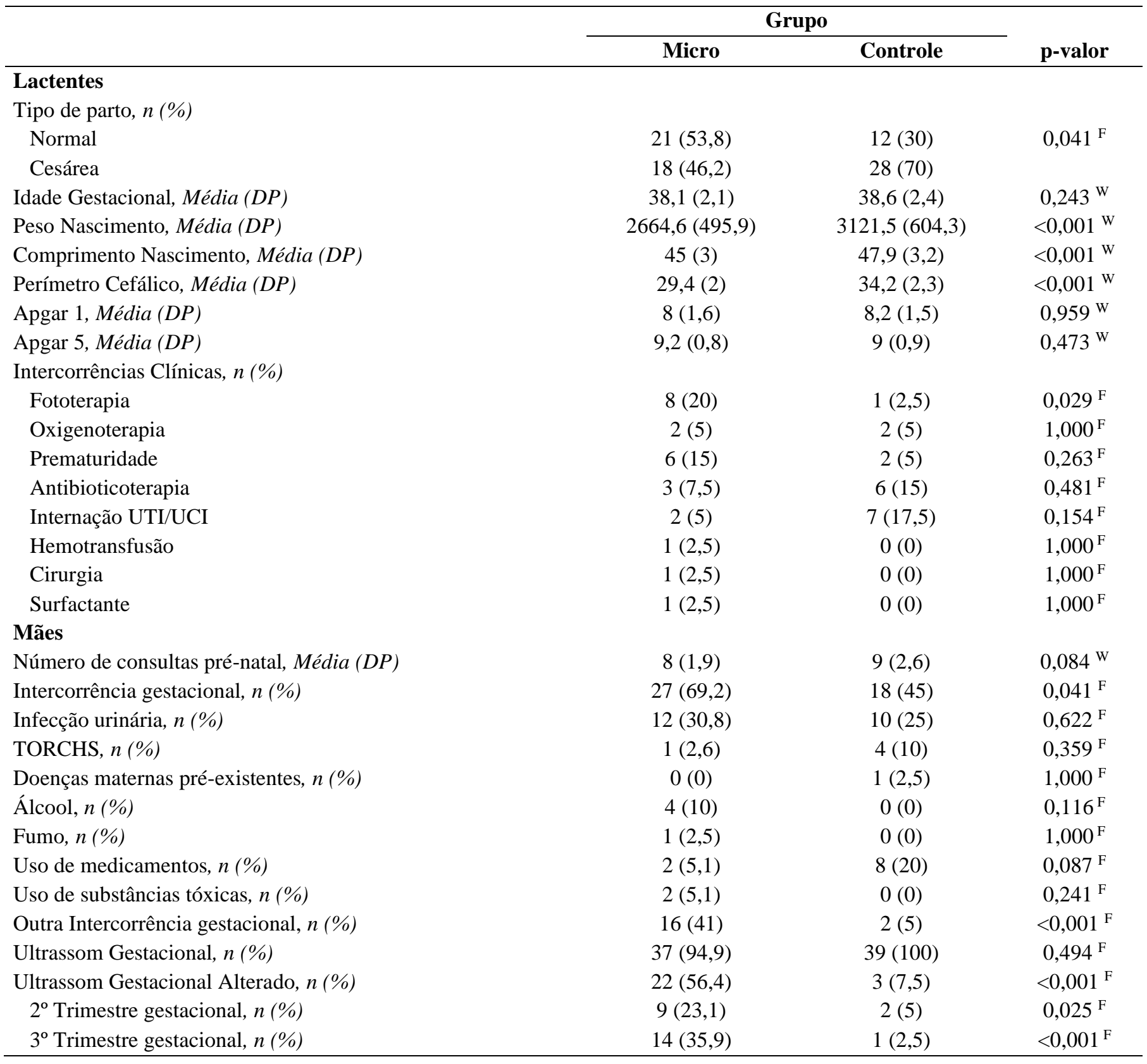

Legenda: $\mathrm{n}$ - frequência absoluta. \% - frequência relativa percentual. DP - desvio padrão. T - Teste T para amostras independentes. W Teste de Mann-Whitney. T - Teste T para amostras independentes. F - Teste Exato de Fisher.

Na classificação da funcionalidade motora grossa dos lactentes do GM, observou-se que $85 \%$ foram classificados no nível V, $12,5 \%$ no nível IV, e $2,5 \%$ no nível II.

Fonte: Autores. 
Para a análise dos dados do teste AHEMD, foram excluídos cinco indivíduos do GM por não terem concluído o seu preenchimento, mesmo após a assinatura do TCLE. Sendo assim, foram analisados nesse teste, comparativamente, 75 lactentes, distribuídos em 35 do GM e 40 do GC.

As oportunidades de estimulação para o desenvolvimento motor apresentaram diferenças significativas nas dimensões variedade de estimulação, brinquedo motricidade fina e pontuação total onde o GM apresentou oportunidades menos adequadas. Com relação ao desempenho motor grosso, houve diferenças significativas em todas as dimensões do GMFM-88, onde o GM obteve menor pontuação quando comparado com o grupo controle (Tabela 3).

Tabela 3. Descrição do desempenho do GM e GC nas avaliações do AHEMD e do GMFM.

\begin{tabular}{|c|c|c|c|}
\hline & & & \\
\hline & Micro & Controle & p-valor \\
\hline AHEMD & & & \\
\hline Espaço Físico, Média (DP) & $1,5(1,3)$ & $2,5(1,8)$ & $0,011 \mathrm{~W}$ \\
\hline Espaço Físico, $n(\%)$ & & & \\
\hline Menos adequado & $23(65,7)$ & $18(45)$ & $0,010^{Q}$ \\
\hline Moderadamente adequado & $12(34,3)$ & $11(27,5)$ & \\
\hline Adequado & $0(0)$ & $9(22,5)$ & \\
\hline Excelente & $0(0)$ & $2(5)$ & \\
\hline Variedade Estimulação, Média (DP) & $10,7(3,2)$ & $13,1(4)$ & $0,001 \mathrm{~W}$ \\
\hline Variedade Estimulação, $n(\%)$ & & & \\
\hline Menos adequado & $16(45,7)$ & $2(5)$ & $<0,001 \mathrm{Q}$ \\
\hline Moderadamente adequado & $8(22,9)$ & $3(7,5)$ & \\
\hline Adequado & $5(14,3)$ & $17(42,5)$ & \\
\hline Excelente & $6(17,1)$ & $18(45)$ & \\
\hline Brinquedo Motricidade Grossa, Média (DP) & $4,6(1,7)$ & $5,6(3,3)$ & $0,091^{\mathrm{T}}$ \\
\hline Brinquedo Motricidade Grossa, $n(\%)$ & & & \\
\hline Menos adequado & $31(88,6)$ & $22(55)$ & $0,002 \mathrm{Q}$ \\
\hline Moderadamente adequado & $4(11,4)$ & $4(10)$ & \\
\hline Adequado & $0(0)$ & $13(32,5)$ & \\
\hline Excelente & $0(0)$ & $1(2,5)$ & \\
\hline Brinquedo Motricidade Fina, Média (DP) & $3,8(2,6)$ & $7,3(5)$ & $0,002 \mathrm{~W}$ \\
\hline Brinquedo Motricidade Fina, $n(\%)$ & & & \\
\hline Menos adequado & $28(80)$ & $16(40)$ & $<0,001 \mathrm{Q}$ \\
\hline Moderadamente adequado & $6(17,1)$ & $5(12,5)$ & \\
\hline Adequado & $1(2,9)$ & $14(35)$ & \\
\hline Excelente & $0(0)$ & $5(12,5)$ & \\
\hline Pontuação Total, Média (DP) & $20,7(5,5)$ & $28,6(10,5)$ & $<0,001^{\mathrm{T}}$ \\
\hline Pontuação Total, $n(\%)$ & & & \\
\hline Menos adequado & $27(77,1)$ & $8(20)$ & $<0,001 \mathrm{Q}$ \\
\hline Moderadamente adequado & $8(22,9)$ & $13(32,5)$ & \\
\hline Adequado & $0(0)$ & $10(25)$ & \\
\hline Excelente & $0(0)$ & $9(22,5)$ & \\
\hline GMFM & & & \\
\hline Pontuação Percentual Total GMFM-88 & $11,2(7,6)$ & $48,8(11,8)$ & $<0,001 \mathrm{w}$ \\
\hline Pontuação Percentual GMFM-88 - A & $35,6(20,2)$ & $68,8(10,5)$ & $<0,001^{\mathrm{T}}$ \\
\hline Pontuação Percentual GMFM-88 - B & $14,8(13,4)$ & $63,7(11,5)$ & $<0,001 \mathrm{w}$ \\
\hline Pontuação Percentual GMFM-88 - C & $2,6(8)$ & $40,8(17)$ & $<0,001 \mathrm{w}$ \\
\hline Pontuação Percentual GMFM-88 - D & $0,2(0,7)$ & $46(20,4)$ & $<0,001 \mathrm{w}$ \\
\hline Pontuação Percentual GMFM-88 - E & $0(0,2)$ & $23,7(11,6)$ & $<0,001 \mathrm{w}$ \\
\hline
\end{tabular}

Legenda: $\mathrm{n}$ - frequência absoluta. \% - frequência relativa percentual. DP - desvio padrão. T - Teste T para amostras independentes. W Teste de Mann-Whitney. T - Teste T para amostras independentes. Q - Teste Qui-Quadrado de Pearson com simulações de Monte-Carlo. Fonte: Autores. 
Na tabela 4 apresentam-se os modelos múltiplos simples e ajustados para os testes AHMED e GMFM para idade gestacional ao nascimento, tipo de parto, intercorrências clínicas, idade materna, renda familiar, número de crianças e adultos no domicílio e ocupação da mãe. Observa-se que os modelos ajustados são válidos pois não há violação dos pressupostos de autocorrelação ( $\mathrm{DW} \approx 2$ ), multicolinearidade (VIF<5) e nenhum ponto de influência ou outlier $\left(\mathrm{D}^{\max }<1\right)$. Contudo, ao se avaliar a qualidade do ajuste, AHMED possui uma baixa qualidade de ajuste $\left(\mathrm{R}^{2}=0,376\right)$, enquanto o modelo para GMFM se apresenta com uma boa qualidade de ajuste $\left(\mathrm{R}^{2}=0,850\right)$.

Com relação aos parâmetros significativos obtidos, observa-se que para AHMED, em ambos os modelos a escala apresentam pontuações próximas, implicando que as variáveis podem não explicar a pontuação na escala. Para a escala GMFM, observa-se que o primeiro modelo em relação ao modelo ajustado, a escala aumenta para 39,02 pontos, e que as variáveis têm um peso significativo, sendo um modelo explicador dessa pontuação.

Tabela 4. Regressão Linear Múltipla para GMFCS e AHMED ajustado para Microcefalia.

\begin{tabular}{|c|c|c|c|c|c|c|c|c|}
\hline & B & EP & $\mathbf{B}_{\text {pdr }}$ & $\mathbf{T}$ & $\mathbf{p}$ & IC95\%- & IC95\%+ & VIF \\
\hline \multicolumn{9}{|l|}{ AHMED } \\
\hline Intercepto & $-0,043$ & 0,309 & & $-0,139$ & 0,890 & $-0,659$ & 0,574 & \\
\hline Micro/Controle & 1,271 & 0,192 & 0,613 & 6,626 & $<0,001$ & 0,889 & 1,654 & \\
\hline \multicolumn{9}{|c|}{$\mathrm{R}^{2}=0,376 ; \mathrm{R}^{2}$ Ajustado $=0,367 ; \mathrm{DW}=1,835 ; \mathrm{F}=43,90(\mathrm{p}<0,001) ; \mathrm{D}^{\max }=0,043$} \\
\hline \multicolumn{9}{|l|}{ AHMED } \\
\hline Intercepto & 2,918 & 2,385 & & 1,223 & 0,223 & $-1,875$ & 7,771 & \\
\hline Micro/Controle (Ajustado) & 1,214 & 0,259 & 0,560 & 4,689 & $<0,001$ & 0,694 & 1,735 & 1,895 \\
\hline \multicolumn{9}{|c|}{$\mathrm{R}^{2}=0,631 ; \mathrm{R}^{2}$ Ajustado $=0,525 ; \mathrm{DW}=2,313 ; \mathrm{F}=5,975(\mathrm{p}<0,001) ; \mathrm{D}^{\max }=0,391$} \\
\hline \multicolumn{9}{|c|}{ e } \\
\hline Intercepto & $-26,295$ & 3,502 & & $-7,508$ & $<0,001$ & $-33,267$ & $-19,323$ & \\
\hline Micro/Controle & 37,539 & 2,215 & 0,887 & 16,948 & $<0,001$ & 33,129 & 41,949 & \\
\hline \multicolumn{9}{|c|}{$\mathrm{R}^{2}=0,786 ; \mathrm{R}^{2}$ Ajustado $=0,784 ; \mathrm{DW}=1,887 ; \mathrm{F}=287,226(\mathrm{p}<0,001) ; \mathrm{D}^{\max }=0,112$} \\
\hline \multicolumn{9}{|c|}{ 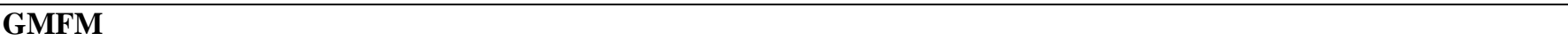 } \\
\hline Intercepto & $-103,897$ & 30,816 & & $-3,372$ & 0,001 & $-165,824$ & $-41,970$ & \\
\hline Micro/Controle (Ajustado) & 39,02 & 3,346 & 0,888 & 11,662 & $<0,001$ & 32,297 & 45,745 & 1,895 \\
\hline \multicolumn{9}{|c|}{$\mathrm{R}^{2}=0,850 ; \mathrm{R}^{2}$ Ajustado $=0,807 ; \mathrm{DW}=2,285 ; \mathrm{F}=19,834(\mathrm{p}<0,001) ; \mathrm{D}^{\max }=0,181$} \\
\hline $\begin{array}{l}\text { Legenda: B - Coeficiente da R } \\
\text { T. IC95\% - Limite inferior do } \\
\text { de Inflação da Variância. DW } \\
\text { gestacional ao nascimento, tip } \\
\text { ocupação da mãe. Fonte: Autol }\end{array}$ & $\begin{array}{l}\text { P - erro pac } \\
\text { com } 95 \% \text { de } \\
\text { Durbin-Wa } \\
\text { intercorrênc }\end{array}$ & $\begin{array}{l}\text { lo. } B_{\text {pdr }}- \\
\text { onfiança. } \\
\text { on. F - Es } \\
\text { clínicas }\end{array}$ & $\begin{array}{l}\text { eficiente } \\
995 \%+- \\
\text { tística } F \\
\text { taca }\end{array}$ & $\begin{array}{l}\text { adronizac } \\
\text { imite sup } \\
\text { Smax }- \text { Ma }^{\text {a }}\end{array}$ & $\begin{array}{l}\text { da Regre } \\
\text { or do int }\end{array}$ & $\begin{array}{l}\text {. } \mathrm{T}-\text { Estati } \\
\text { lo com } 95 \%\end{array}$ & $\begin{array}{l}\mathrm{T} . \mathrm{p}-\mathrm{P}- \\
\text { confiança }\end{array}$ & $\begin{array}{l}\text { do teste } \\
\text { - Fatol } \\
\text { ra: idade } \\
\text { micílio e }\end{array}$ \\
\hline
\end{tabular}

\section{Discussão}

Este estudo descreve o desempenho e as oportunidades motoras de lactentes com microcefalia por ZIKV comparado a lactentes saudáveis. Também demonstra que a maioria dos lactentes com microcefalia por ZIKV apresentam grave comprometimento da função motora grossa sendo a maior parte classificado no nível V do GMFCS, assim como relatada em outros estudos (A. Ventura et al., 2020; Einspieler et al., 2019; Melo et al., 2020). As diferenças evidenciadas no desempenho e oportunidades de estimulação entre os grupos apontam a microcefalia como um fator de risco importante para graves atrasos no desenvolvimento motor.

Com relação as questões clínicas de nascimento, as diferenças observadas entre os grupos para peso, comprimento e perímetro cefálico, cujos valores apresentaram-se menores no GM, corroboram com as características de nascimento descritas dessa população (Cabral et al., 2017; Frota et al., 2020; Melo et al., 2020). As variáveis gestacionais maternas aqui apresentadas também confirmam com os resultados de outros estudos que descrevem a presença dos sintomas do ZIKV no 
primeiro trimestre de gestação (Frota et al., 2020; Melo et al., 2020). Entretanto, os grupos deste estudo apresentaram diferenças importantes para dados sociodemográficos, embora provenientes das mesmas instituições públicas de saúde, tais como região de residência, renda familiar, estado civil e ocupação materna, se apresentaram sempre desfavoráveis no GM.

Sabe-se que a epidemia do Zika vírus impactou significativamente as crianças e famílias da região nordeste (Ministério da Saúde, 2020), que historicamente já apresentam condições menos favoráveis de saúde, educação, econômica e social, cujos estados concentram os menores índices de desenvolvimento humano, apresentando diferenças importantes entre suas microrregiões (Brasil. Instituto de Pesquisa Econômica Aplicada (Ipea) et al., 2016). A literatura (Cabral et al., 2017; Cruz et al., 2017) mostra que em Sergipe e Alagoas a maioria das crianças e suas famílias são do interior dos estados, onde predominam baixas condições socioeconômicas, com mães jovens, solteiras, sem renda fixa e que têm menor tempo de escolaridade. Além disso, a maioria das cidades do interior com incidência de casos de SCZ não dispõe de serviços especializados para prestar assistência, necessitando de deslocamentos dessa população para as capitais (Félix \& Farias, 2018), reforçando a necessidade de uma rede de atenção integral para atender as necessidades das crianças e famílias acometidas pelo ZIKV.

As oportunidades de estimulação motoras no domicílio foram menos adequadas no GM, nas dimensões variedade de estimulação, brinquedo motricidade fina e pontuação total do AHEMD. Estudos (Bezerra, 2019; Zancanelli, 2018) realizados em outros estados também evidenciaram baixas oportunidades de estimulação motora para lactentes com microcefalia por ZIKV. Zancanelli 2018, ao avaliar 14 lactentes com idades entre oito e 20 meses em Juiz de Fora e região, o autor relatou baixas oportunidades de estimulação no ambiente domiciliar e relações moderadas a fortes entre essas affordances e o desenvolvimento motor, sendo classificadas como menos ou moderadamente adequado para a maioria dos lactentes. Bezerra 2019, ao avaliar 30 crianças com microcefalia entre 18 e 42 meses em duas cidades do Rio Grande do Norte, encontrou a maioria famílias com vulnerabilidade socioeconômica, poucas oportunidades de estimulação motora e baixo desempenho cognitivo.

Analisando as características das famílias, embora não haja diferenças entre o tipo de moradia e escolaridade materna entre os grupos; houve diferenças significativas para idade materna, ocupação e renda familiar, com valores sempre menores no GM em relação ao GC. Esses dados poderiam explicar as dificuldades enfrentadas pelas famílias do GM em proporcionar ambientes favoráveis para o desenvolvimento motor. Conforme relatado em outro estudo (Cabral et al., 2017), características como menor idade materna, desemprego e menor renda, implicam na necessidade em investimentos para reduzir os efeitos sociais negativos vivenciados por essas mulheres, e a garantia de assistência à saúde e social integral por meio políticas públicas. A renda (Soares et al., 2015) tem sido considerada como decisiva para que as famílias tenham qualidade de vida e acesso a serviços relacionados à saúde, educação e habitação.

O GMFM-88 foi utilizado para avaliar o desempenho motor grosso dos lactentes por ser a versão mais indicada para crianças com grave comprometimento motor, contendo uma versão mais detalhada das habilidades motoras atuais da criança classificadas no nível V do GMFCS (Russel et al., 2015). Os resultados mostram que os participantes do GM apresentaram menores pontuações em comparação ao GC em todas as dimensões do GMFM-88, e suas maiores habilidades motoras concentram-se nas dimensões A e B, o que implica afirmar que são compatíveis com lactentes com idade cronológica até 6 meses ou inferior.

Outros estudos foram feitos nesse sentido, e corroboram com nossos achados; Melo et al. 2020 avaliou 59 crianças com SCZ com idades entre 5 a 29 meses utilizando o GMFM-88 e o GMFCS e evidenciou que $81 \%$ apresentou grave comprometimento da função motora, sendo que o escore percentual do GMFM teve mediana de 6,5,com intervalo interquartil 4,2, e apenas 4 crianças pontuaram nas dimensões D e E. Frota et al. 2020 avaliou 50 crianças com SCZ aos 24 meses de idade utilizando o GMFM-88 e evidenciou que comorbidades apresentaram associações significativas nas dimensões A e B do teste, 
sendo menores na presença de epilepsia e disfagia. A. Ventura et al. 2020 acompanhou 77 lactentes com suspeita de infeção pelo ZIKV nos 2 primeiros anos de vida, utilizando o GMFM-66 e o GMFCS, e evidenciou que todas as crianças com diagnóstico para paralisia cerebral apresentaram grave comprometimento da motricidade grossa.

Os achados do presente estudo, bem como os demais estudos levantados (A. Ventura et al., 2020; Bezerra, 2019; Marques et al., 2019; Melo et al., 2020; Zancanelli, 2018), sobre o comprometimento do desempenho motor grosso e a deficiência nas oportunidades de estimulação domiciliar de sujeitos com microcefalia por ZIKV, apontam para a necessidade de acompanhamento sistemático dessa população a fim de otimizar as oportunidades para estimulação do desenvolvimento, o que deve ser tido como um enfrentamento a ser realizado pelos profissionais de saúde à luz de políticas públicas efetivas.

O presente estudo evidenciou que a microcefalia apresentou um impacto importante nos desfechos avaliados nos grupos comparativos, tal como em outros estudos (Carvalho et al., 2019; Marques et al., 2019) que têm demonstrado o grave comprometimento das habilidades motoras de crianças com microcefalia por ZIKV, associando perímetro cefálico e malformações corticais graves a pior função motora. Este estudo contribui para entender sobre o desempenho e oportunidades de estimulação motora de lactentes com microcefalia por ZIKV, de dois estados específicos do nordeste brasileiro, comparando-os com lactentes saudáveis.

Preconiza-se, nesse sentido, o desenvolvimento de programas, ações e estruturação de serviços centrados na família, para que estas possam propiciar ambientes mais ricos em oportunidades de estimulação para o desenvolvimento infantil (Folha et al., 2018). A proposição de intervenções com foco em ambientes naturais, como o domiciliar, pode ser uma estratégia de intervenção eficiente e de baixo custo (Cunha et al., 2018; Novak et al., 2017).

\section{Conclusão}

Lactentes com microcefalia por ZIKV apresentam grave comprometimento do desempenho motor grosso e baixas oportunidades de estimulação motora. Os resultados deste estudo reforçam a necessidade de uma assistência integral, centrada na família, uma vez que a resolutividade dos impactos do ZIKV sobre o desenvolvimento motor e o cuidado prestado pelas famílias é uma questão de saúde pública importante.

\section{Referências}

A. Ventura, P., C. Lage, M.-L., L. de Carvalho, A., S. Fernandes, A., B. Taguchi, T., \& Nascimento-Carvalho, C. M. (2020). Early Gross Motor Development Among Brazilian Children with Microcephaly Born Right After Zika Virus Infection Outbreak. Journal of Developmental \& Behavioral Pediatrics, 41(2), 134-140. https://doi.org/10.1097/DBP.0000000000000722

Baud, D., Gubler, D. J., Schaub, B., Lanteri, M. C., \& Musso, D. (2017). An update on Zika virus infection. The Lancet, 390(10107), 2099-2109. https://doi.org/10.1016/S0140-6736(17)31450-2

Bezerra, D. A. (2019). Affordances motoras no ambiente domiciliar de crianças com microcefalia e o desempenho cognitivo [Universidade Federal do Rio Grande do Norte]. https://repositorio.ufrn.br/jspui/handle/123456789/27349

Brasil. Instituto de Pesquisa Econômica Aplicada (Ipea), Programa das Nações Unidas para o Desenvolvimento (PNUD), \& Fundação João Pinheiro (FJP). (2016). Desenvolvimento humano nas macrorregiões brasileiras. IPEA. PNUD. FJP. http://repositorio.ipea.gov.br/bitstream/11058/6217/1/Desenvolvimento humano nas macrorregiões brasileiras.pdf

Brasil. Ministério da Saúde. Secretaria de Atenção à Saúde. (2016). Diretrizes de estimulação precoce: crianças de zero a 3 anos com atraso no desenvolvimento neuropsicomotor. Ministério da Saúde. http://portalarquivos.saude.gov.br/images/pdf/2016/novembro/26/Diretrizes-de-estimulacaoprecoce.pdf

Brasil. Ministério da Saúde. Secretaria de Vigilância em Saúde. (2020). Monitoramento dos casos de arboviroses urbanas transmitidas pelo Aedes Aegypti (dengue, chikungunya e zika), Semanas Epidemiológicas 1 a 16, 2020. Boletim Epidemiológico, 51(17), 1-47. https://www.saude.gov.br/images/pdf/20 20/April/24/Boletim-epidemiologico-SVS-17-.pdf Acesso em: 11.Ago.2020.

Brunoni, D., Blascovi-Assis, S. M., Osório, A. A. C., Seabra, A. G., Amato, C. A. de la H., Teixeira, M. C. T. V., da Rocha, M. M., \& Carreiro, L. R. R. (2016). Microcephaly and other Zika virus related events: The impact on children,families and health teams. Ciencia e Saude Coletiva, 21 (10), 3297-3302. https://doi.org/10.1590/1413-812320152110.16832016 
Cabral, C. M., Nóbrega, M. E. B. da, Leite, P. L. e, Souza, M. S. F. de, Teixeira, D. C. P., Cavalcante, T. F., Lima, R. G. de S., Tavares, L. M. S. de A., Souza, P. B. de, Saad, E., Cabral, C. M., Nóbrega, M. E. B. da, Leite, P. L. e, Souza, M. S. F. de, Teixeira, D. C. P., Cavalcante, T. F., Lima, R. G. de S., Tavares, L. M. S. de A., Souza, P. B. de, \& Saad, E. (2017). Descrição clínico-epidemiológica dos nascidos vivos com microcefalia no estado de Sergipe, 2015. Epidemiologia e Serviços de Saúde, 26(2), 245-254. https://doi.org/10.5123/S1679-49742017000200002

Caçola, P. M., Gabbard, C., Montebelo, M. I. L., \& Santos, D. C. C. (2015). The new affordances in the home environment for motor development - infant scale (AHEMD-IS): Versions in English and Portuguese languages. Brazilian Journal of Physical Therapy, 19(6), 507-525. https://doi.org/10.1590/bjptrbf. 2014.0112

Carvalho, A., Brites, C., Mochida, G., Ventura, P., Fernandes, A., Lage, M. L., Taguchi, T., Brandi, I., Silva, A., Franceschi, G., Lucena, P., \& Lucena, R. (2019). Clinical and neurodevelopmental features in children with cerebral palsy and probable congenital Zika. Brain and Development, 41(7), 587-594. https://doi.org/10.1016/j.braindev.2019.03.005

Conselho Nacional de Saúde. (2013). Resolução n 466, de 12 de dezembro de 2012. Aprova diretrizes e normas regulamentadoras de pesquisas envolvendo seres humanos. Diário Oficial Da União. http://conselho.saude.gov.br/resolucoes/2012/Reso466.pdf

Cruz, T. A. R. da, Silva, F. C. da, \& Santos, E. M. de S. (2017). Perfil clínico, sociodemográfico e funcional dos lactentes com microcefalia atendidos no setor de Terapia Ocupacional de um Centro Especializado em Reabilitação. Reunião Anual Da SBPC, 69, 1-4. http://www.sbpcnet.org.br/livro/69ra/resumos/resumos/3120_16fe617d671a2801bacd0298f53c577a1.pdf

Cunha, A. B., Miquelote, A. F., \& Santos, D. C. C. (2018). Motor affordance at home for infants living in poverty: A feasibility study. Infant Behavior and Development, 51, 52-59. https://doi.org/10.1016/j.infbeh.2018.03.002

del Campo, M., Feitosa, I. M. L., Ribeiro, E. M., Horovitz, D. D. G., Pessoa, A. L. S., França, G. V. A., García-Alix, A., Doriqui, M. J. R., Wanderley, H. Y. C., Sanseverino, M. V. T., Neri, J. I. C. F., Pina-Neto, J. M., Santos, E. S., Verçosa, I., Cernach, M. C. S. P., Medeiros, P. F. V., Kerbage, S. C., Silva, A. A., van der Linden, V., \& Schuler-Faccini, L. (2017). The phenotypic spectrum of congenital Zika syndrome. American Journal of Medical Genetics Part A, 173(4), 841-857. https://doi.org/10.1002/ajmg.a.38170

Duarte, J. dos S., Santos, L. O. F., Sette, G. C. S., Santos, T. de F. C., Alves, F. A. P., \& Coriolano-Marinus, M. W. de L. (2019). Necessidades de crianças com síndrome congênita pelo Zika vírus no contexto domiciliar. Cadernos Saúde Coletiva, 27(3), 249-256. https://doi.org/10.1590/1414-462x201900030237

Einspieler, C., Utsch, F., Brasil, P., Panvequio Aizawa, C. Y., Peyton, C., Hydee Hasue, R., Françoso Genovesi, F., Damasceno, L., Moreira, M. E., Adachi, K., Marschik, P. B., \& Nielsen-Saines, K. (2019). Association of Infants Exposed to Prenatal Zika Virus Infection With Their Clinical, Neurologic, and Developmental Status Evaluated via the General Movement Assessment Tool. JAMA Network Open, 2(1), e187235. https://doi.org/10.1001/jaman etworkopen.2018.7235

Félix, V. P. da S. R., \& Farias, A. M. de. (2018). Microcefalia e dinâmica familiar: a percepção do pai frente à deficiência do filho. Cadernos de Saúde Pública, 34(12). https://doi.org/10.1590/0102-311x00220316

Folha, D. R. da S. C., Marini, B. P. R., Nunes, A. C., \& Barba, P. C. de S. Della. (2018). Terapia Ocupacional e a atenção a crianças com Síndrome Congênita do Zika Vírus na perspectiva da Intervenção Precoce. Revista Argentina de Terapia Ocupacional, 1(4), 30-39. http://www.revista.terapiaocupacional.org.ar/RATO/2018jul-art4.pdf

Fox, J., \& Weisberg, S. (2018). An R Companion to Applied Regression. Sage publications.

Freitas, T. C. B., Gabbard, C., Caçola, P., Montebelo, M. I. L., \& Santos, D. C. C. (2013). Family socioeconomic status and the provision of motor affordances in the home. Brazilian Journal of Physical Therapy, 17(4), 319-327. https://doi.org/10.1590/S1413-35552013005000096

Frota, L. M. da C. P., Sampaio, R. F., Miranda, J. L., Brasil, R. M. C., Gontijo, A. P. B., Mambrini, J. V. de M., Brandão, M. de B., \& Mancini, M. C. (2020). Children with congenital Zika syndrome: symptoms, comorbidities and gross motor development at 24 months of age. Heliyon, 6(6), e04130. https://doi.org/10.1016/j.heliyon.2020.e04130

Gabbard, C., Caçola, P., \& Rodrigues, L. P. (2008). A New Inventory for Assessing Affordances in the Home Environment for Motor Development (AHEMD-SR). Early Childhood Education Journal, 36(1), 5-9. https://doi.org/10.1007/s10643-008-0235-6

Heymann, D. L., Hodgson, A., Sall, A. A., Freedman, D. O., Staples, J. E., Althabe, F., Baruah, K., Mahmud, G., Kandun, N., Vasconcelos, P. F. C., Bino, S., \& Menon, K. U. (2016). Zika virus and microcephaly: Why is this situation a PHEIC? The Lancet, 387(10020), 719-721. https://doi.org/10.1016/S01406736(16)00320-2

Kapogiannis, B. G., Chakhtoura, N., Hazra, R., \& Spong, C. Y. (2017). Bridging Knowledge Gaps to Understand How Zika Virus Ex posure and Infection Affect Child Development. JAMA Pediatrics, 171(5), 478. https://doi.org/10.1001/jamapediatrics.2017.0002

Marques, F. J. P., Teixeira, M. C. S., Barra, R. R., de Lima, F. M., Dias, B. L. S., Pupe, C., Nascimento, O. J. M., \& Leyser, M. (2019). Children Born With Congenital Zika Syndrome Display Atypical Gross Motor Development and a Higher Risk for Cerebral Palsy. Journal of Child Neurology, 34(2), 81-85. https://doi.org/10.1177/0883073818811234

Melo, A., Gama, G. L., Da Silva Júnior, R. A., De Assunção, P. L., Tavares, J. S., Da Silva, M. B., Costa, K. N. F. S., Vânia, M. L., Evangelista, M. A., \& De Amorim, M. M. R. (2020). Motor function in children with congenital Zika syndrome. Developmental Medicine \& Child Neurology, 62(2), 221-226. https://doi.org/10.1111/dmcn.14227

Ministério da Saúde. Secretaria de Vigilância. (2020). Boletim Epidemiológico. Situação epidemiológica da síndrome congênita associada à infecção pelo vírus Zika em 2020, até a SE 45 (No. 05; Vol. 51). http://plataforma.saude.gov.br/anomalias-congenitas/boletim-epidemiologico-SVS-05-2020.pdf

Novak, I., Morgan, C., Adde, L., Blackman, J., Boyd, R. N., Brunstrom-Hernandez, J., Cioni, G., Damiano, D., Darrah, J., Eliasson, A.-C., de Vries, L. S., Einspieler, C., Fahey, M., Fehlings, D., Ferriero, D. M., Fetters, L., Fiori, S., Forssberg, H., Gordon, A. M., ... Badawi, N. (2017). Early, Accurate Diagnosis and Early Intervention in Cerebral Palsy. JAMA Pediatrics, 171(9), 897. https://doi.org/10.1001/jamapediatrics.2017.1689 
Research, Society and Development, v. 10, n. 7, e7110716347, 2021

(CC BY 4.0) | ISSN 2525-3409 | DOI: http://dx.doi.org/10.33448/rsd-v10i7.16347

Pessoa, A., van der Linden, V., Yeargin-Allsopp, M., Carvalho, M. D. C. G., Ribeiro, E. M., Van Naarden Braun, K., Durkin, M. S., Pastula, D. M., Moore, J. T., \& Moore, C. A. (2018). Motor Abnormalities and Epilepsy in Infants and Children With Evidence of Congenital Zika Virus Infection. Pediatrics, 141(Supplement 2), S167-S179. https://doi.org/10.1542/peds.2017-2038F

Russel, D. J., Rosenbaum, P. L., Wright, M., \& Avery, L. M. (2015). Medida da Função Motora Grossa (GMFM-66 \& GMFM-88): Manual do usuário. (Tradução Luara Tomé Ciryllo e Maria Cristina dos Santos Galvão) (Segunda Ed). Memnon.

Santos, D. B. C. dos, Silva, E. F. da, Lima, S. O., Reis, F. P., \& Oliveira, C. da C. C. (2019). Health Care Network: Maternal perception regarding the quality of care to children with microcephaly. Escola Anna Nery, 23(4). https://doi.org/10.1590/2177-9465-ean-2018-0335

Silva, D. B. R., Pfeifer, L. I., \& Funayama, C. A. R. (2010). GMFCS - E\&R: Sistema de classificação da função motora grossa- ampliado e revisto. https://canchild.ca/system/tenon/assets/attachments/000/000/075/original/GMFCS-ER_Translation-Portuguese2.pdf

Soares, E. S., Flores, F. S., Katzer, J. I., Valentini, N. C., Corazza, S. T., \& Copetti, F. (2015). Análise das oportunidades de estimulação motora em ambientes domiciliares na região central do Rio Grande do Sul. Revista Brasileira de Educação Física e Esporte, 29(2), 279-288. https://doi.org/10.1590/180755092015000200279

Zancanelli, A. M. (2018). Infecção gestacional por Zika vírus: desenvolvimento motor nos primeiros anos de vida, contexto ambiental e perfil epidemiológico. Universidade Federal de Juiz de Fora. 